\title{
Medical Image Recognition of Abdominal Organs by RBF GMDH-type Neural Network
}

\author{
Tadashi Kondo ${ }^{1)}$ and Junji Ueno \\ School of Health Sciences, The University of Tokushima, 3-18-15 Kuramoto-cho \\ Tokushima 770-8509, Japan
}

E-mail: kondo@medsci.tokushima-u.ac.jp ${ }^{1)}$

\begin{abstract}
A Radial Basis Function (RBF) Group Method of Data Handling (GMDH)-type neural network algorithm proposed in this paper is applied to the medical image recognition of abdominal X-ray CT images. The optimum neural network architecture for the medical image recognition is automatically organized using RBF GMDH-type neural network algorithm and the regions of abdominal organs such as the liver, stomach and spleen are recognized and extracted accurately.
\end{abstract}

\section{Introduction}

The conventional GMDH-type neural networks [1],[2] are automatically organized using the heuristic self-organization method [3],[4] and the structural parameters such as the number of layers, the number of neurons in each layer, useful input variables and optimum neuron architectures are automatically determined so as to minimize the prediction error criterion Akaike's Information Criterion (AIC) [5] or Prediction Sum of Squares (PSS) [6]. In this paper, a RBF GMDH-type neural network algorithm is proposed. In this algorithm, the polynomial type and the RBF type neurons are used for organizing the neural network architecture. A lot of complex nonlinear combinations of the input variables fitting the complexity of the nonlinear system are generated using the polynomial type neurons and only useful combinations of the input variables are selected for organizing the neural network architecture. In the output layer, the RBF type neuron is used for organizing the neural network and the output value of the neural network becomes between zero and one.

In the medical image processing, there are many kinds of medical images such as a multi-detector row computed tomography (MDCT) image, magnetic resonance imaging (MRI) image, X-ray image and mammography, whose image characteristics are different each other. When we apply the neural network to medical image recognition of medical images and extract the interested regions of the organs, we must organize the neural network architectures fitting the characteristics of the medical images, but it is very different to find the optimum neural network architecture fitting the characteristics of the medical image because the optimum neural network architectures are different in many kinds of the medical images and the organs. The RBF GMDH-type neural network is applied to the medical image recognition of the abdominal X-ray CT images and it is shown that the RBF GMDH-type neural network is accurate and useful method for the medical image recognition of the abdominal organs because the optimum neural network architecture is automatically organized.

\section{Heuristic Self-Organization}

The GMDH-type neural network algorithm can automatically develop the optimum neural network architectures by the heuristic self-organization. The heuristic self-organization in the GMDH-type neural network is implemented through the following five procedures:

\section{1) Separating the original data into training and test sets.}

The original data are separated into training and test sets. The training data are used for the estimation of the weights of the neural network. The test data are used for organizing neural network architectures.

2) Generating the combinations of the input variables in each layer.

All combinations of $r$ input variables are generated in each layer. The number of combinations is $\frac{p !}{(p-r) ! r !}$. Here, $p$ is the number of input variables and the number of $r$ is usually set to two.

\section{3) Selecting the optimum neuron architectures}

For each combination, the optimum neuron architectures which describe the partial characteristics of the nonlinear system can be calculated by applying the regression analysis [7] to the training data. The output variables $y_{k}$ of the optimum neurons are called intermediate variables. In the GMDH-type neural network, the optimum neurons are selected from different neuron architectures. 
4) Selecting the intermediate variables.

The $L$ intermediate variables which give the $L$ smallest test errors calculated using the test data are selected from the generated intermediate variables $y_{k}$. Selected $L$ intermediate variables are set to the input variables of the next layer and calculations from procedure 2 to 4 are iterated.

5) Stopping the multilayered iterative computation.

When the errors for the test data in each layer stop decreasing, the iterative computation is terminated. The complete neural network which describes the characteristics of the nonlinear system can be constructed using the optimum neurons which are generated in each layer.

\section{RBF GMDH-Type Neural Network Algorithm}

The RBF GMDH-type neural network architecture is shown in Fig.1. Here, nonlinear function $g_{i}$ is described by the following Kolmogorov-Gabor polynomial:

$$
g_{i}\left(x_{1}, x_{2}, \cdots, x_{p}\right)=a_{0}+\sum_{i} a_{i} x_{i}+\sum_{i} \sum_{j} a_{i j} x_{i} x_{j}+\cdots
$$

This nonlinear function is automatically organized by using the polynomial neurons. The architecture of the RBF GMDH-type neural network is automatically organized using the heuristic self-organization and is produced as follows:

In the RBF GMDH-type neural network, the original data are not separated into training and test sets because PSS can be used as the test errors.

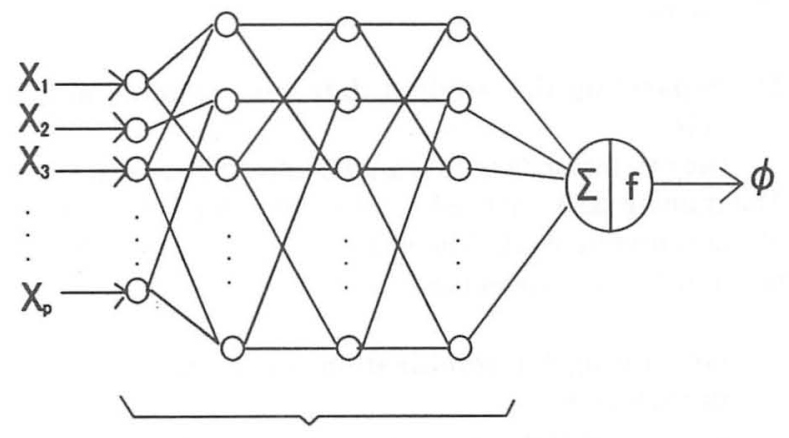

$$
g_{i}\left(x_{1}, x_{2}, \cdots, x_{p}\right)
$$

$\Sigma:$ (Nonlinear function)

$$
z_{k}=\sum w_{i} g_{i}\left(x_{1}, x_{2}, \cdots, x_{p}\right)
$$

$f:$ (Nonlinear function)

$$
\phi=\exp \left(-z_{k}^{2}\right)
$$

Fig.1 Architecture of RBF GMDH-type neural network

\subsection{The first layer}

$$
u_{j}=x_{j} \quad(j=1,2, \cdots, p)
$$

where $x_{j}(j=1,2, \cdots, p)$ are the input variables of the system, and $p$ is the number of input variables. In the first layer, input variables are set to the output variables.

\subsection{The second layer}

Many combinations of two variables $\left(u_{i}, u_{j}\right)$ are generated. For each combination, the neuron architecture is described by the following equations:

$$
\begin{aligned}
& \sum \text { : (Nonlinear function) } \\
& z_{k}=w_{1} u_{i}+w_{2} u_{j}+w_{3} u_{i} u_{j}+w_{4} u_{i}^{2}+w_{5} u_{j}^{2}+w_{6} u_{i}^{3}+w_{7} u_{i}^{2} u_{j} \\
& \quad+w_{8} u_{i} u_{j}^{2}+w_{9} u_{j}^{3}-w_{0} \theta_{l} \\
& \text { f: (Linear function) } \\
& \quad y_{k}=z_{k}
\end{aligned}
$$

where $\theta_{1}=1$ and $w_{i}(i=0,1,2, \cdots, 9)$ are weights between the first and second layer. The weights $w_{i}(i=0,1,2, \cdots, 9)$ are estimated by using the multiple regression analysis. This procedure is as follows:

First, the values of $z_{k}$ are calculated using the following equation:

$$
z_{k}=\left(-\log _{e} \phi^{\prime}\right)^{1 / 2}
$$

where $\phi^{\prime}$ is the normalized output variable. Then the weights $w_{i}(i=0,1,2, \cdots, 9)$ are estimated by using the stepwise regression analysis which selects useful input variables using the PSS. Therefore, only useful variables in (3) are selected and neuron architecture can be organized by these selected useful variables.

From these generated neurons, $L$ neurons which minimize PSS values are selected. The output values $\left(y_{k}\right)$ of $L$ selected neurons are set to the input values of the neurons in the third layer.

\subsection{The third and succeeding layers}

In the third and succeeding layers, the same computation of the second layer is continued until PSS values of $L$ neurons are not decreased. When the iterative computation is terminated, the following calculation of the output layer is carried out.

\subsection{The output layer}

In the output layer, the output values of the neural network are calculated from $z_{k}$ as follows:

$$
y_{k}=\exp \left(-z_{k}^{2}\right)
$$

So, in the output layer, the neuron architecture becomes as follows:

$\sum$ : (Nonlinear function)

$$
\begin{gathered}
z_{k}=\sum w_{i} g_{i}\left(x_{1}, x_{2}, \cdots, x_{p}\right) \\
f:(\text { Nonlinear function) } \\
\phi=\exp \left(-z_{k}^{2}\right)
\end{gathered}
$$

At last, the complete neural network architecture is produced by selected neurons in each layer.

Figure 2 shows the flowchart of the RBF GMDH- type neural network. 


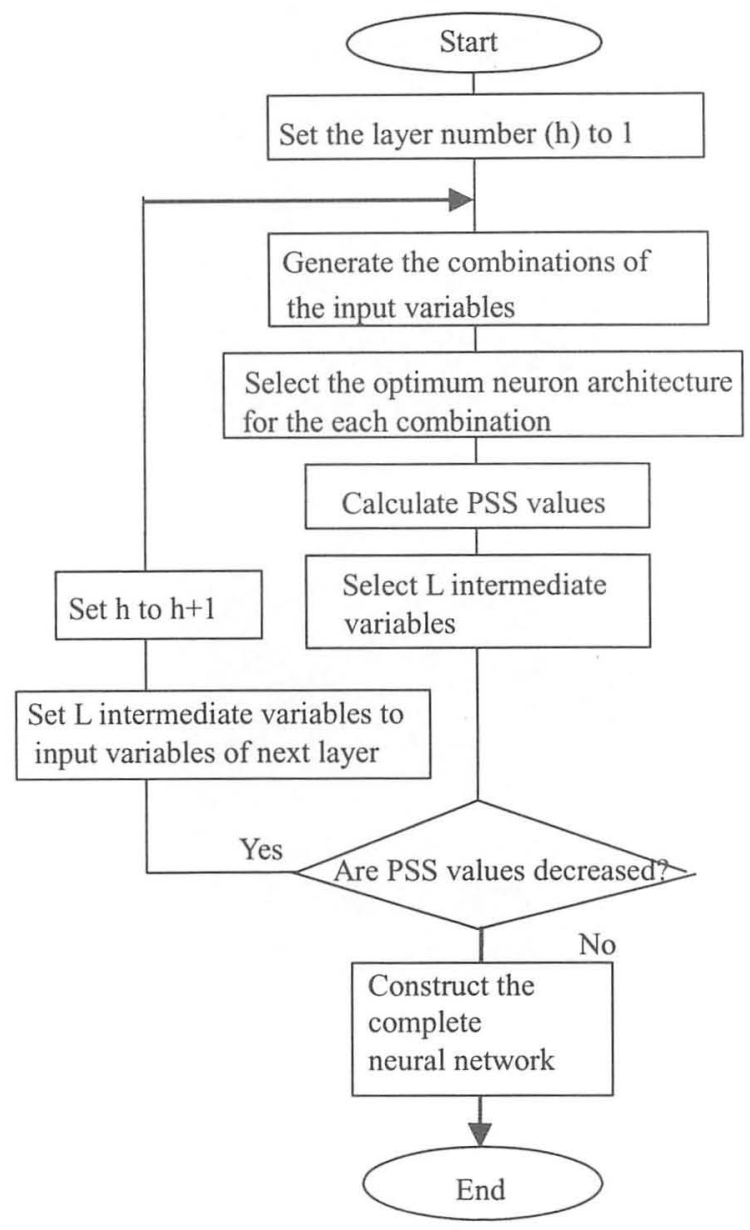

Fig. 2 Flowchart of the RBF GMDH-type neural network

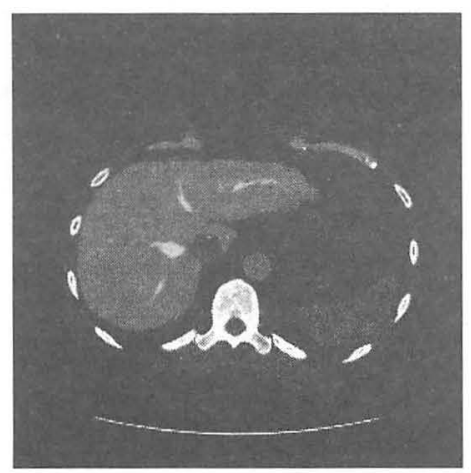

Fig.3 Original image

\section{Application to Medical Image Recognition}

In this study, the regions of the liver, stomach and spleen were recognized and extracted automatically using the RBF GMDH-type neural network. Medical images used in this study are abdominal X-ray CT images. The pixel values of the liver, stomach and spleen regions are very similar each other and therefore, it is very difficult to recognize and extract accurately the regions of these organs using the artificial neural networks.

\subsection{Extraction of the liver region}

Figure 3 shows one of the original images which were used for organizing the RBF GMDH-type neural network. The statistics of the image densities in the neighboring regions, the $\mathrm{N} \times \mathrm{N}$ pixel regions, were used as the image features. Three statistics, mean, standard deviation and variance, were used as the input variables and other input variables were $\mathrm{x}, \mathrm{y}$ and $\mathrm{z}$ coordinates of 3-dimensional position of the learning points. In the reference [2], statistics of image densities in neighboring regions, $\mathrm{N} \times \mathrm{N}$ pixel regions, were used as image features and the following statistics were used as input variables of the neural network. 1) mean, 2) standard deviation, 3) variance, 4) median, 5) minimum, 6) maximum, 7) range. Out of those statistics, only three parameters namely, mean, standard deviation and variance were selected as useful input variables. Therefore, in this study, we used these three statistics as the useful input variables of the neural network. Furthermore, $x, y$ and $\mathrm{z}$ coordinates of 3-dimensional position of the learning points are important as the input variables and so these variables are used in this study. The output value of the neural network was zero or one. When $\mathrm{N} \times \mathrm{N}$ pixel region was contained in the region of the liver, the neural network set the pixel value at the center of the $\mathrm{N} \times \mathrm{N}$ pixel region to one and this pixel was shown as the white point. The neural networks were organized when the values of $\mathrm{N}$ were from 3 to 10 . It was determined that when $\mathrm{N}$ was equal to 5 , the neural network architecture had the smallest recognition error. Five useful neurons were selected in each hidden layer. Figure 4 shows the variation of PSS values. In this figure, open circles show the PSS values of the neurons in each layer and solid lines show the combinations of two variables $\left(x_{i}, x_{j}\right)$ in each layer. The calculation of the RBF GMDH-type neural network terminated at the sixth layer. PSS values at the second layer were not small but they decreased and became very small one at the sixth layer because the architecture of the RBF GMDH-type neural network became more complex and fit the complexity of the medical image characteristics. From Fig.4, the PSS values of the neurons are different each other between second and fourth layers but, PSS values converged in the sixth layer. As the results, the calculation of the GMDH-type neural network terminated because five neurons in the sixth layer has almost the same characteristics concerned with the prediction error. If the calculation of the GMDH-type neural network is continued over the sixth layer, the generalization ability can not be improved. The RBF GMDH-type neural network output the liver image and the post-processing analysis of the liver image was carried out, based on which the region of the liver was extracted. Figure 5 shows the output image of the RBF GMDH-type neural network. Figure 6 shows the output image after the post-processing. In the 
post-processing of the output image, the small isolated regions were eliminated and the outlines of the liver regions were expanded outside by $\mathrm{N} / 2$ pixels. The overlapped image is shown in Fig.7. The recognized liver region is very accurate. The liver region was extracted from the original image using the output image. Figure 8 shows the extracted image of the liver.

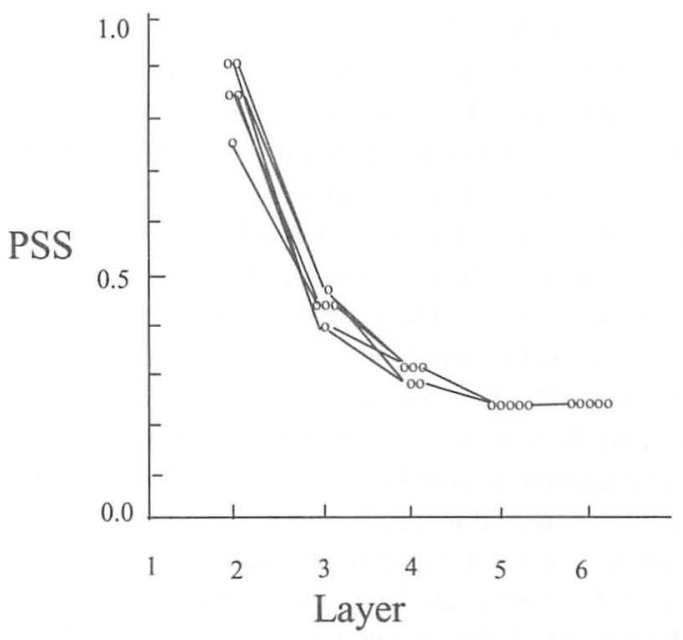

Fig.4 Variation of PSS values (1)

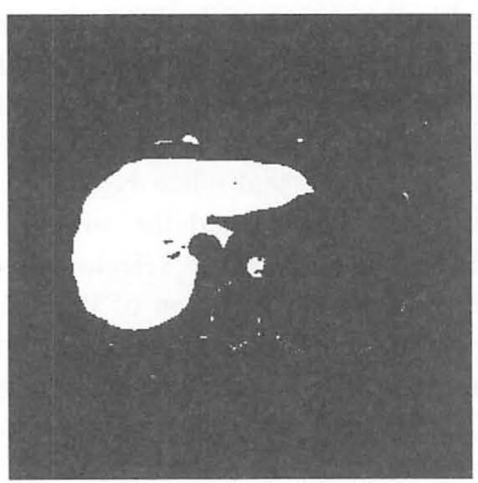

Fig.5 Output image(1)

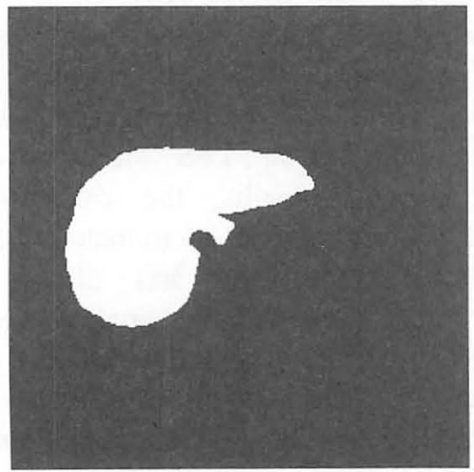

Fig.6 Output image after post-processing(1)

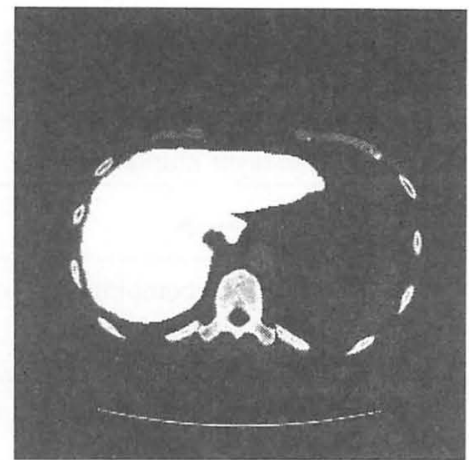

Fig.7 Overlapped image(1)

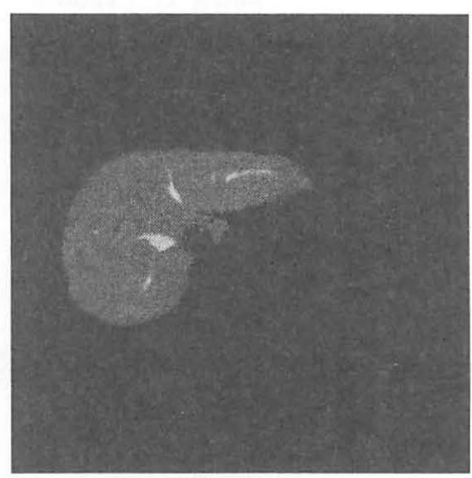

Fig. 8 Extraction of the liver region

\subsection{Extractions of the stomach region}

New other RBF GMDH-type neural network was organized to recognize and extract the regions of the stomach. The organization procedures of the neural network are the same as those of the liver. Figure 9 shows the variation of PSS values. The calculation of the RBF GMDH-type neural network also terminated at the sixth layer and very small PSS values were obtained. The recognition results of the stomach are shown in from Fig.10 to 13. The regions of the stomach were also recognized and extracted very accurately.

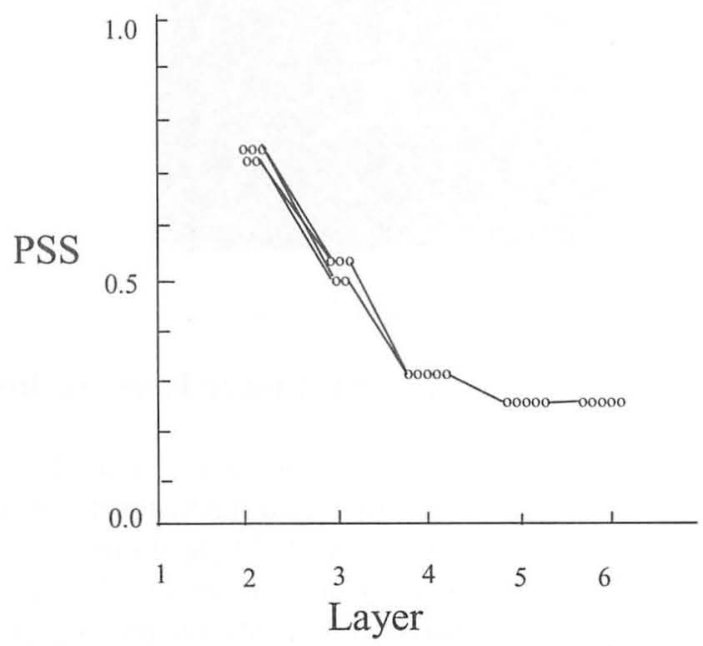

Fig.9 Variation of PSS values (2) 


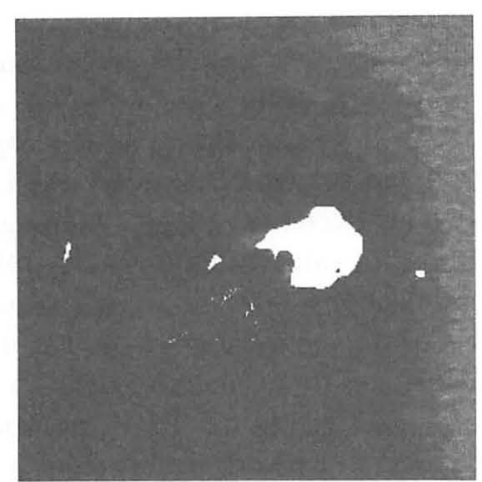

Fig.10 Output image (2)

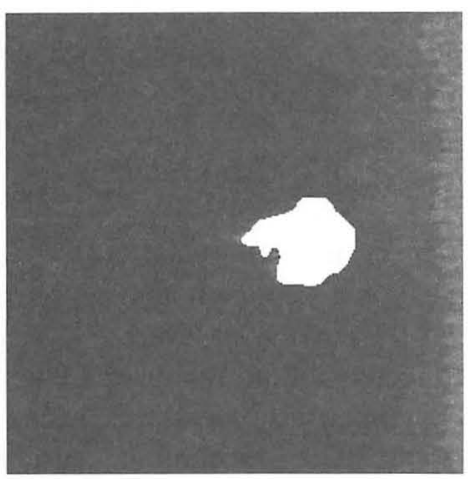

Fig.11 Output image after post-processing (2)

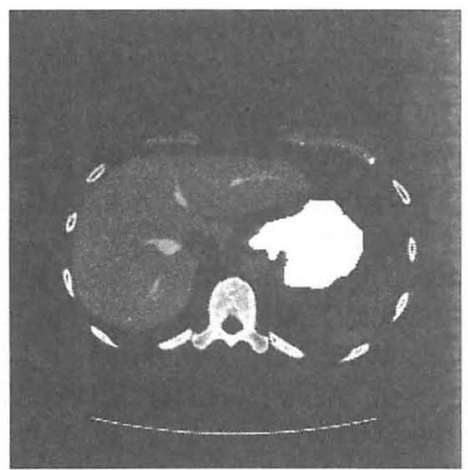

Fig.12 Overlapped image (2)

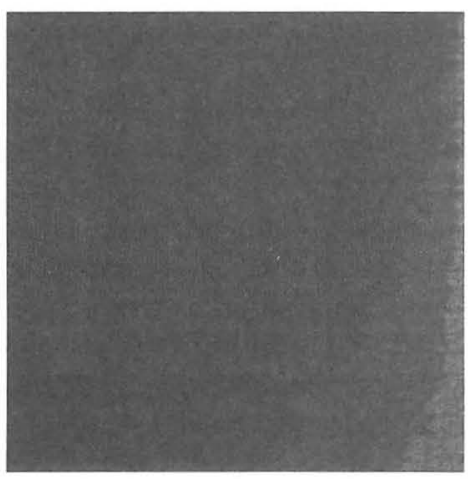

Fig.13 Extraction of the stomach region

\subsection{Extractions of the spleen region}

New other RBF GMDH-type neural network was organized to recognize and extract the regions of the spleen. The organization procedures of the neural network are the same as those of the liver and stomach. Figure 14 shows the variation of PSS values. The calculation of the RBF GMDH-type neural network also terminated at the sixth layer and very small PSS values were obtained. The recognition results of the spleen are shown in from Fig.15 to 18 . The regions of the spleen were also recognized and extracted very accurately.

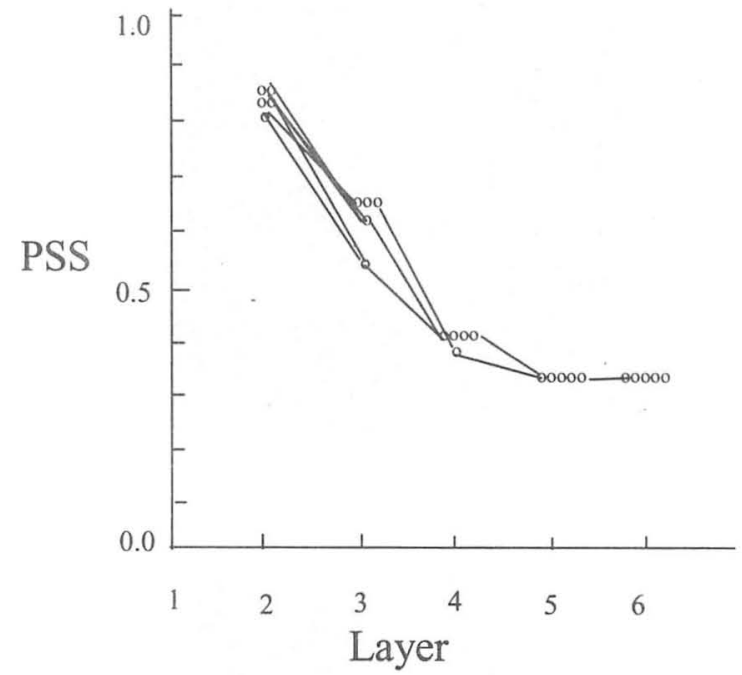

Fig.14 Variation of PSS values (3)

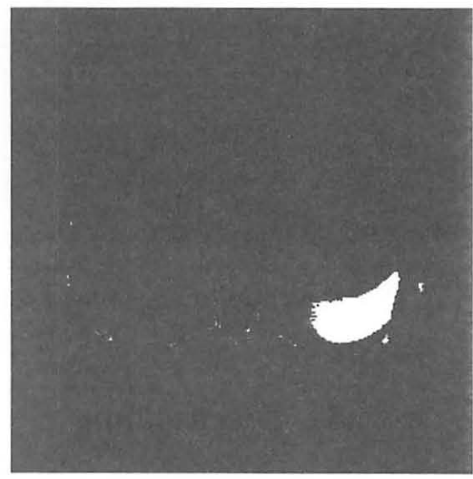

Fig.15 Output image (3) 


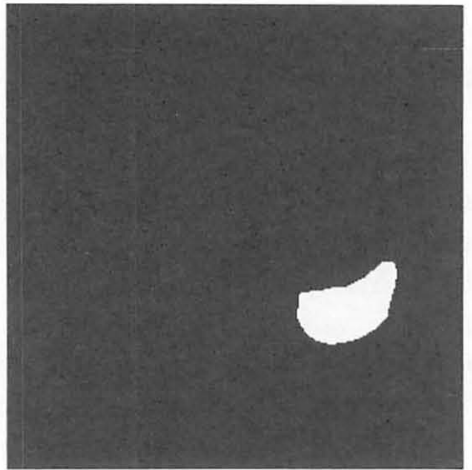

Fig.16 Output image after post-processing (3)

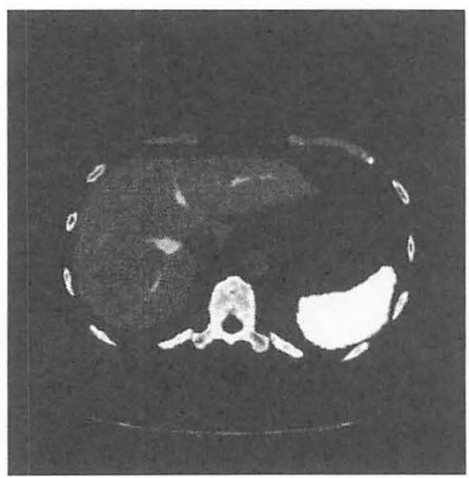

Fig.17 Overlapped image(3)

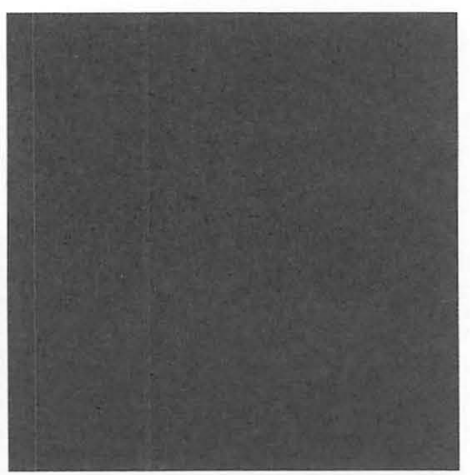

Fig.18 Extraction of the spleen region

\section{Discussions}

Medical images used in this study are abdominal X-ray CT images. The pixel values of the liver, stomach and spleen regions are very similar each other and therefore, it is very difficult to recognize and extract accurately the regions of these organs using the artificial neural networks. The RBF GMDH-type neural network proposed in this paper is applied to the medical image recognitions of the liver, stomach and spleen. In the RBF GMDH-type neural network algorithm, many complex nonlinear combinations of the input variables fitting the complexity of the nonlinear system are generated and only useful combinations of the input variables are selected for organizing the neural network architecture using the prediction error criterion defined as PSS. In the medical image recognition of the liver, stomach and spleen, PSS values at the second layer were not small but decreased gradually through many layers and very small PSS values were obtained at the sixth layer. In these calculations of the layers, many nonlinear combinations of the input variables are generated and only useful combinations fitting the complexity of the medical images were selected using PSS criterion. The RBF GMDH-type neural network architecture was organized using these selected nonlinear combinations of the input variables. Output images of the RBF GMDH-type neural network are very accurate and the regions of the liver, stomach and spleen are distinguished and recognized clearly using the RBF GMDH-type neural network.

\section{Conclusions}

In this paper, the RBF GMDH-type neural network algorithm was proposed and applied to medical image recognition of the abdominal X-ray $\mathrm{CT}$ image. The neural network architecture fitting the complexity of the medical image was automatically organized using the heuristic self-organization. The recognition results were very accurate and it was shown that this algorithm was a useful method of the medical image recognition.

\section{References}

[1] T.kondo, J.Ueno and K.Kondo,"Revised GMDH-type neural networks using AIC or PSS criterion and their application to medical image recognition", JACIII, Vol.9, No.3, pp.257-267, 2005.

[2] T.Kondo, J.Ueno and A.S.Pandya, "Multilayered GMDH-Type Neural Network with Radial Basis Functions and its Application to 3-Dimensional Medical Image Recognition of the Liver", JACIII, Vol.11, No.1,pp.96-104,2007

[3] A.G.Ivakhnenko, "Heuristic self-organization in problems of engineering cybernetics", Automatica, Vol.6, No.2, pp.207-219, 1970.

[4] S.J.Farlow Ed., Self-organizing Methods in Modeling, GMDH-type Algorithms, New York, Marcel Dekker, Inc., 1984.

[5] H.Akaike, "A new look at the statistical model identification", IEEE Trans. Automatic Control, Vol.AC-19, No.6, pp.716-723, 1974.

[6] H.Tamura, and T.Kondo, "Heuristics free group method of data handling algorithm of generating optimum partial polynomials with application to air pollution prediction", INT. J. SYSTEMS SCI., Vol 11, No.9, pp.1095-1111, 1980.

[7] N.R.Draper, and H.Smith, Applied Regression Analysis, New York, John Wiley and Sons, 1981. 